\title{
Simultaneous Temperature and Strain Discrimination in a Conventional BOTDA via Artificial Neural Networks
}

\author{
Ruben Ruiz-Lombera, Alberto Fuentes, Luis Rodriguez-Cobo, Jose Miguel Lopez-Higuera, Fellow, OSA Senior \\ Member, IEEE and Jesus Mirapeix
}

\begin{abstract}
A system based on the use of Artificial Neural Networks (ANNs) allowing discrimination of strain and temperature in a conventional Brillouin Optical Time Domain Analyzer (BOTDA) setup is presented and demonstrated in this paper. This solution allows to perform an automatic discrimination of both parameters without compromising the complexity or cost of the interrogation unit. The classification results, achieved by considering a pre-processing stage with dimensionality reduction via Principal Component Analysis (PCA) and spatial filtering, improve those obtained in a previous feasibility study.
\end{abstract}

Index Terms-Optical fiber sensors, distributed systems, stimulated Brillouin scattering, strain-temperature discrimination, artifical neural network

\section{INTRODUCTION}

O PTICAL fiber distributed sensors have experienced an intense research effort in the last years. Stimulated Brillouin scattering (SBS) based systems, particularly via Brillouin Optical Time Domain Analyzer (BOTDA) implementations, are one of the most popular solutions due to their ability to provide both temperature and strain measurements over long sensing ranges (several tens of kilometers) and with typical spatial resolutions of about 1 meter. Their performance has been greatly improved in different areas. The sensing range has been extended up to $100 \mathrm{~km}$ by means of coding techniques [1] or Raman [2] or Brillouin assistance [3]. A $325 \mathrm{~km}$ BOTDA with four EDFA (erbium doped fiber amplifier) repeaters has also been demonstrated [4]. The spatial resolution, limited in standard BOTDA configurations to the $1 \mathrm{~m}$ limit, has been improved using different approaches such as the differential pulse-pair technique [5], but also via Brillouin Optical Frequency Domain Analyzer (BOFDA) and Brillouin Optical Correlation Domain Analyzer (BOCDA) systems. The limitations imposed on both pump and probe signal input powers have also been studied and overcome with various strategies [6], [7]. Dynamic measurements have also been

R. Ruiz-Lombera, A. Fuentes, J.M. Lopez-Higuera and J. Mirapeix are with the Photonics Engineering Group, University of Cantabria (Spain) email: (jesus.mirapeix@unican.es).

L. Rodriguez-Cobo is with Biomedical Research Networking Center in Bioengineering Biomaterials and Nanomedicine (CIBER-BBN), Cantabria (Spain)

J.M. Lopez-Higuera and J. Mirapeix are also with Biomedical Research Networking Center in Bioengineering Biomaterials and Nanomedicine (CIBER-BBN), Cantabria (Spain) and Instituto de Investigacion Sanitaria Valdecilla (IDIVAL), Cantabria (Spain).

Manuscript received XXXXX X, 201X; revised ... . achieved with techniques such as slope-assisted BOTDA [8] or more sophisticated approaches [9], [10].

Given the high amount of contributions, in an attempt to allow a normalized comparison of the performance of these different approaches, a figure-of-merit was proposed in [11], where the uncertainty in the determination of the Brillouin frequency shift (BFS), and therefore in the estimation of temperature and/or strain, is demonstrated to be directly related to the signal-to-noise ratio (SNR) at the receiver. Recently, some processing strategies have been proposed to improve the associated SNR, for example via $2 \mathrm{D}$ and 3D image restoration techniques [12]. Artificial intelligence strategies, in particular based on artificial neural networks (ANNs), have also been proposed to perform the BFS estimation [13].

Within this framework, some efforts are still being devoted to overcome one of the fundamental limitations of conventional BOTDA implementations: their inability to perform a simultaneous discrimination between temperature/strain measurements. In these systems, temperature and strain estimations are performed via BFS, as there is a known linear relation between this frequency shift and the temperature/strain applied to a particular section of the deployed fiber [14]. This similar behavior of the BFS makes it difficult to distinguish the contributions of each parameter to the final frequency shift. A simple solution, in fact the first proposed to solve this problem, is based on the use of a second sensing optical fiber, deployed to be insensitive to strain [15]. The simultaneous employment of Raman or Rayleigh-based [16] distributed systems in addition to a BOTDA may be a possible solution, although at the expense of increasing the complexity and cost of the final setup. Some authors have also proposed the use of large-effective-area nonzero-dispersion-shifted (LEAF) [17] or photonic crystal (PCF) [18] fibers. In these cases several peaks with different temperature and strain dependences appear in the Brillouin spectra, allowing the desired discrimination. Recently, discrimination of temperature and strain in distributed measurements has been achieved by means of a system combining standard coherent optical time-domain reflectrometry and Rayleigh-based distributed birefringence measurements in an elliptical-core polarization-maintaining fiber [19]. A dual-BOTDA scheme has also been proposed, 
where the simultaneous measurement of temperature and strain is performed using the different wavelength scaling factor of temperature and strain coefficients at 850 and 1550 $\mathrm{nm}[20]$.

These proposals typically imply the use of more complex systems or special fibers, thus affecting the cost of the resulting systems. In this paper, we propose the use of a conventional BOTDA system, including a standard monomode fiber as fiber-under-test (FUT), where an artificial intelligence solution, in particular an artificial neural network (ANN), is used to process the BOTDA data and perform the requested discrimination. Although the temperature and strain resolutions considered in our solution are still clearly lower than those obtained in previous works, the resulting BOTDA setup complexity and its associated cost would remain unaltered. Distributed simultaneous temperature and strain measurements will demonstrate the feasibility of the proposed solution.

\section{THEORY}

As previously stated, in a BOTDA system there is a linear relation between the BFS and the estimated temperature and strain, which can be expressed as follows [21]:

$$
v_{B}(\delta T, \delta \epsilon)-v_{B 0}=A \cdot \delta \epsilon+B \cdot \delta T,
$$

where $v_{B}$ is the BFS, $v_{B 0}$ the Brillouin frequency of the chosen optical fiber at room temperature $\left(T_{0}=25^{\circ} \mathrm{C}\right), \delta T$ and $\delta \epsilon$ the temperature and strain changes at a given location of the fiber and $A$ and $B$ are constants. Apart from this known frequency displacement, there are some subtle changes in the Brillouin gain spectrum (BGS) depending on the parameter under analysis. As detailed by Nickles et al. in [14], the BGS shows a decrease in its linewidth and an increase in the associated Brillouin gain for increasing temperatures. However, an increasing strain does not clearly affect the BGS width, while the resulting gain decreases in this case.

These subtle variations might be difficult to be used for simultaneous temperature/strain measurements in a conventional BOTDA scheme, but in this paper we propose the use of an ANN to perform the requested temperature/strain classification/discrimination. However, some pre-processing strategies have been also considered to adapt the acquired BOTDA data and try to obtain a suitable performance, in an attempt to improve the classification rate achieved in a previous feasibility study [22].

The first step consisted on a normalization of the amplitude of the acquired BGSs, given that it has been assumed that they will not provide useful information for the temperature/strain discrimination, and they might even compromise the system performance. This normalization has been performed in all the experimental tests carried out in this work. A so-called spatial filtering has also been considered to avoid the negative effect of excessive inhomogeneities in the estimated BFS. In particular, spectra of the same class (i.e. associated with the same temperature and strain) with a BFS deviation over $5 \mathrm{MHz}$ have been removed from the dataset. These two processes have always been implemented, but there are some other stages whose influence in the system performance should be evaluated:

- Lorentzian-Gaussian fit: this process, usually employed in BOTDA systems, enables a better estimation of some key spectral features such as the BFS or the BGS linewidth. 251 spectral samples are considered for each frequency sweep.

- Manual selection of parameters: implies the possibility of directly using the estimated BFS and BGS linewidth of each new spectrum after a Lorentzian-Gaussian fitting process.

- Principal Component Analysis (PCA): a common solution to reduce the dimensionality of the data entering an ANN [23], this technique will be explained in more detail in the following.

PCA is a technique used to express a set of variables into a new orthogonal basis, where the new basis vectors are ordered in terms of the variance of the original data, from high to low (i.e. these new vectors are the data directions containing the most relevant information). This transformation implies that the variables of the new dataset are uncorrelated. In our case, the original dataset used as input for the PCA algorithm will be a $m \times n$ matrix $(X)$, where $m$ denotes the number of frequencies in each individual BOTDA spectrum (251) and $n$ the number of observations. The goal of PCA is to build the matrix $W$ such that $Y=W X$, where $Y$ is the resulting transformed data and $W$ represents the linear operation needed to obtain $Y$ from $X$. The process implemented by PCA to obtain $W$ can be summarized as follows:

- Subtract the mean of each row in $X$

- Calculate the covariance matrix $C=X X^{T}=E D E^{T}$, where $D$ is a diagonal matrix containing the eigenvalues of $C$ and $E$ are the eigenvectors of the covariance matrix C.

- Calculate the eigenvectors and eigenvalues of the covariance matrix $C=V D V^{-1}$, where $V$ is a matrix with the eigenvectors of $C$.

- Order the components in $V$ in terms of the contribution of their associated eigenvalues to the total variance of the dataset.

After this last step $W$, the matrix that allows the desired transformation, is obtained as $W=V$. A criterion is now required to establish the number of components (PCs) or columns in $W$ to be used. Equation 2 defines the percentage of variance (information) that is kept after PCA, where $I_{K}$ is this variance, $K$ the number of chosen PCs (eigenvectors) and $\beta_{i}$ the ith eigenvalue:

$$
I_{K}=\frac{\sum_{i=1}^{K} \beta_{i}}{\sum_{i=1}^{m} \beta_{i}} \cdot 100 \%
$$


It is worth noting that PCA has also been used in other BOTDA applications, for example to perform a pattern recognition for the extraction of temperature distributions [24]. With these possibilities of pre-processing the performance of the ANN can be evaluated, although the design of the network may also influence the final performance. ANNs are a computational approach that may solve a wide range of problems. They try to mimic, in a way, the behavior of the human brain, and they are formed by neurons arranged in different layers. A standard implementation is constituted by an input layer, one or two hidden layers and an output layer. This arrange is known as a feedforward implementation, where the input data are transmitted to the hidden layer and then to the output layer.

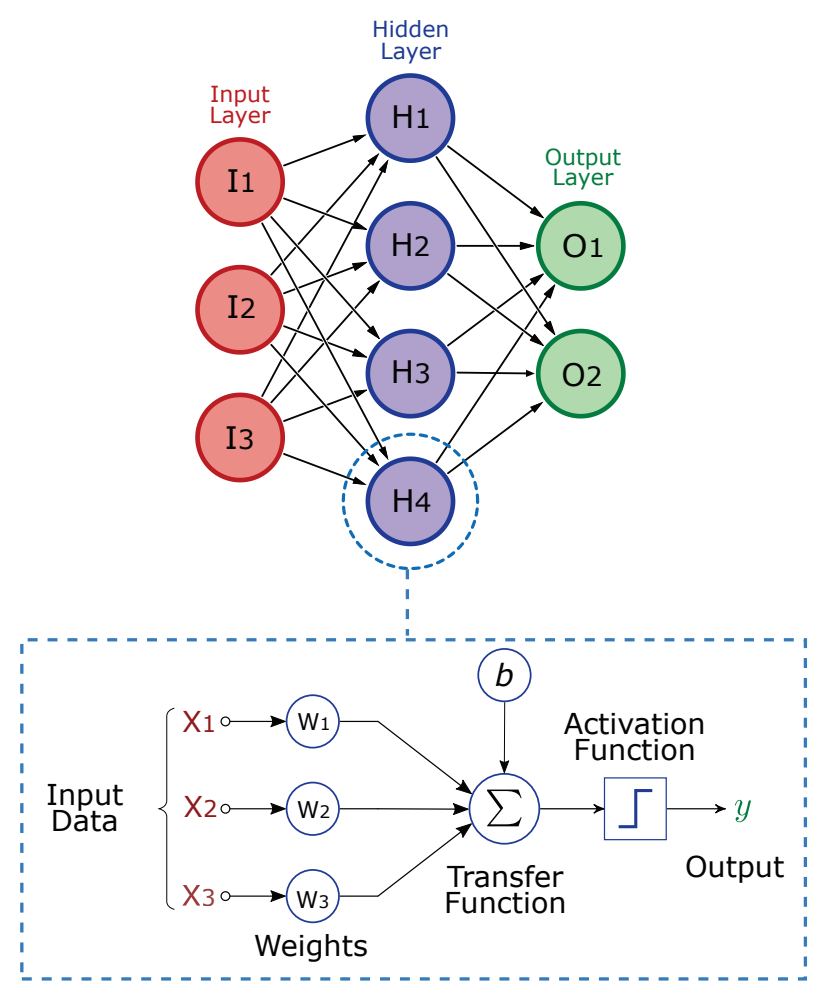

Fig. 1. Schematic representation of an artificial neural network.

In Fig. 1, a simple ANN with 3 input neurons, 4 neurons in a single hidden layer and 2 output neurons has been represented as an example. As can be observed in the inset, a neuron receives several inputs ( $x_{1}$ to $x_{3}$ in the example) that have an associated weight $\left(w_{1}\right.$ to $\left.w_{3}\right)$ indicating the relevance of that input over the output. A weighted combination of inputs is then formed after the transfer function, and the corresponding output will be activated or not depending on the threshold established by the activation function. As ANNs work by activating a specific output neuron for a given input, it is necessary to initially consider a training process where the weights will be determined by means of a given training algorithm. In this stage, the input training data are labeled so that the output neuron to be activated is known. Typically, a $70 \%$ of the whole dataset is devoted to the training process, while the remaining $30 \%$ is equally divided between the validation and test sets. The validation set is used to perform a final tuning of the parameters of the network, for example the number of hidden layers/neurons, but its associated classification rate might be biased as this set is used to select the final ANN model. Consequently, the test set is used only to evaluate the performance of a fully trained network.

It is important to note that two different stages can be considered in the implementation of the proposed solution to the temperature/strain discrimination in a BOTDA system. The first stage (Fig. 2(a)) can be understood as off-line, as it consists in the design and definition of the parameters of both pre-processing techniques and ANN. In our case, the former will be basically devoted to PCA, with the definition of the matrix $W$ to apply the transformation to the original data and the definition of the number of PCs to be used. The most suitable ANN for the problem under analysis and the provided data will be completely defined once the training and validation steps have been performed. The computational cost derived from this stage will not penalize the on-line operation of the final system, schematically represented in Fig. 2(b).

Off-line stage: definition of processing parameters

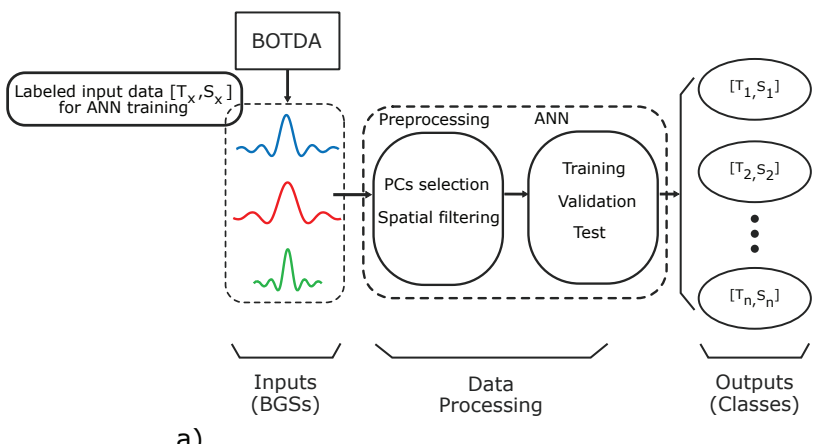

a)

On-line stage: real-time temperature and strain discrimination

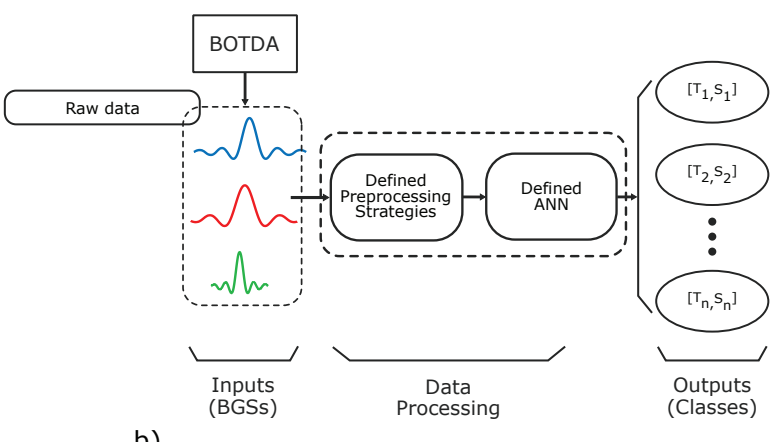

b)

Fig. 2. Schematic representation of the proposed solution: a) off-line stage: definition of pre-processing and ANN parameters and architecture and b) online stage: real-time temperature-strain discrimination.

The specific parameters and ANN design selected to carry out the experimental analysis of the proposed solution will be discussed in Section IV. 


\section{EXPERIMENTAL ISSUES}

The system used for the acquisition of the required measurements was based on a conventional dual probe sideband BOTDA balanced implementation. The setup is depicted in Fig. 3. It can be observed that a conventional system was selected, where a distributed feedback laser (DFB) source working at $\lambda=1550.92 \mathrm{~nm}$ generated the light employed for both pump and probe waves via an optical coupler. A semiconductor optical amplifier (SOA) was used in the upper (pump) branch to obtain high extinction-ratio pulses. An erbium-doped fiber amplifier (EDFA) and a polarization scrambler were also deployed in that branch, while the probe wave was generated via a RF-generator and a electro-optical modulator (EOM). The BOTDA traces were detected with a $125 \mathrm{MHz}$ photodetector and an acquisition card, once the probe signal was filtered with a fiber Bragg grating tuned to analyze only the Stokes component.

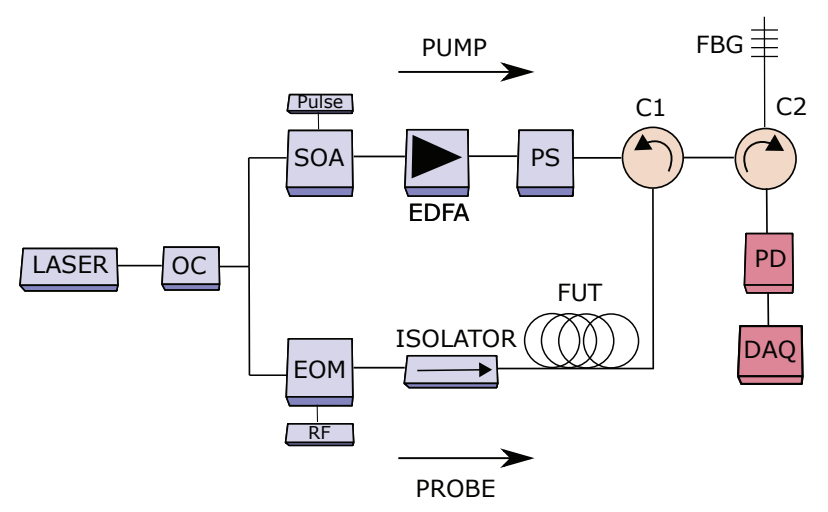

Fig. 3. BOTDA setup employed in the experimental tests: OC (optical coupler); EDFA (erbium-doped fiber amplifier); PS (polarization scrambler); EOM (electro-optical modulator); C (optical circulator); FBG (fiber Bragg grating); PD (photodetector) and DAQ (Acquisition card).

Some initial tests were performed to verify that the hypothesis [14] described in the previous section was correct. In particular, the evolution of the BGS width was analyzed in terms of the temperature for various widths of the chosen pump pulse. Given that a fiber spool was used in this case, a constant tension close to 0 strain could be assumed, although the winding process will imply some residual stress. As can be observed in Fig. 4(a), there is a clear decreasing trend in the BGS width for increasing temperatures from 0 to $80{ }^{\circ} \mathrm{C}$ for all the pump pulse widths considered (from 10 to 100 ns). The tests were carried out using a climatic chamber. These results were obtained using a FUT of around $2.8 \mathrm{~km}$ and performing an average over 1000 BOTDA traces. A similar analysis for increasing strains (considering in this case a constant temperature of $22^{\circ} \mathrm{C}$ ) is presented in Fig. 4(b). In this case, only several tens of meters (about $40 \mathrm{~m}$ ) were homogeneously strained due to the limitations imposed by the strain-setup. This also limited the pump widths to be considered: 10,15 and 20ns. A frequency step of $2 \mathrm{MHz}$ and a Lorentzian-Gaussian profile were considered in this case. The results shown in Fig. 4(b) are in good agreement with those reported by Nickes et al. [14], as no clear trend can
TABLE I

BFS STANDARD DEVIATION $(\sigma)$ [MHZ] FOR DIFFERENT TEMPERATURES AND PUMP PULSE WIDTHS.

\begin{tabular}{c|ccccc} 
& \multicolumn{6}{|c}{ Pulse width [ns] } \\
\hline Temperature $\left[{ }^{\circ} \mathbf{C}\right]$ & $\mathbf{1 0}$ & $\mathbf{1 5}$ & $\mathbf{2 0}$ & $\mathbf{5 0}$ & $\mathbf{1 0 0}$ \\
$\mathbf{0}$ & 0.49 & 0.29 & 0.30 & 0.12 & 0.11 \\
$\mathbf{2 0}$ & 0.24 & 0.44 & 0.16 & 0.13 & 0.20 \\
$\mathbf{4 0}$ & 0.68 & 0.42 & 0.19 & 0.12 & 0.08 \\
$\mathbf{6 0}$ & 0.80 & 0.53 & 0.23 & 0.12 & 0.14 \\
$\mathbf{8 0}$ & 0.44 & 0.43 & 0.56 & 0.14 & 0.12
\end{tabular}

be derived from these tests. It is worth noting that the strain was measured in number of turns of the linear positioner employed to strain the fiber. Each turn corresponds to 384 $\mu$ strains.
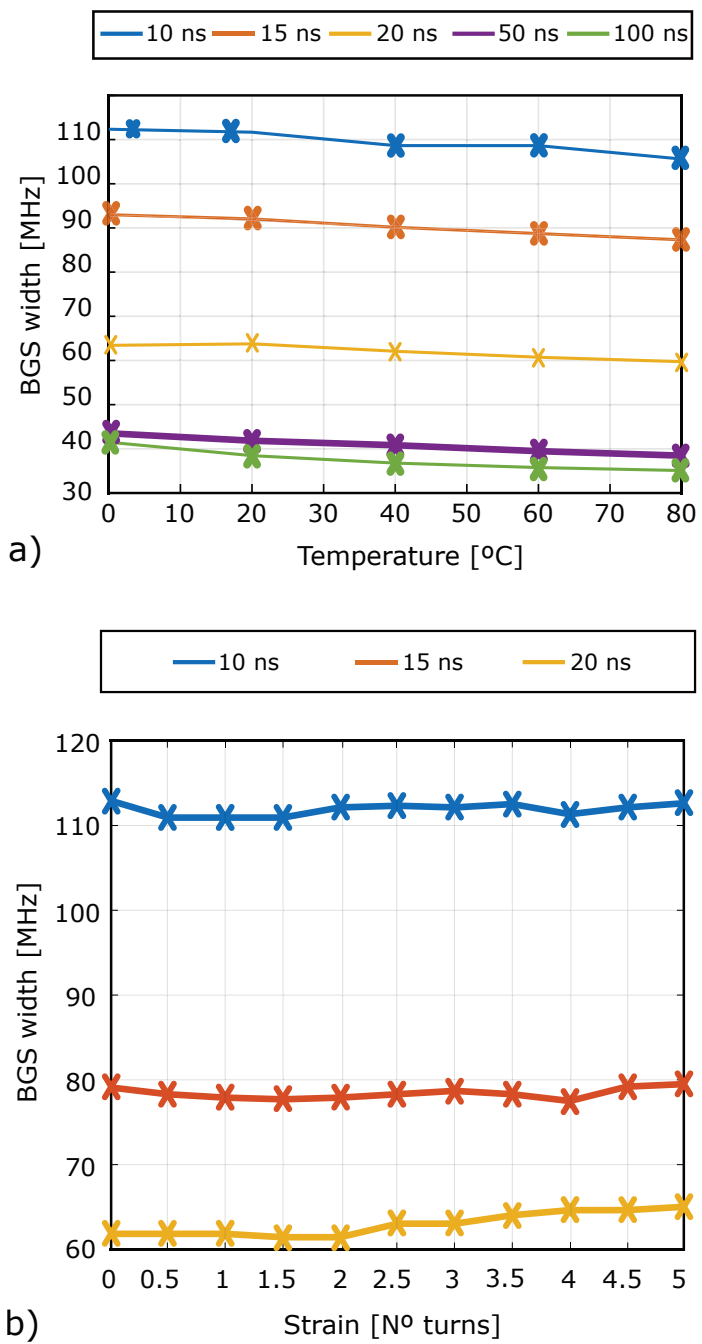

Fig. 4. Evolution of the BGS linewidth for different pump pulse widths for: (a) increasing temperature and (b) increasing strain.

For a further analysis of the acquired data, the BFS standard deviation for each pair of temperature/strain and pump pulse width has been calculated considering 10 consecutive measurements with the same environmental conditions. These results are presented in Tables 1 and 2. 
TABLE II

BFS STANDARD DEVIATION $(\sigma)$ [MHZ] FOR DIFFERENT STRAINS AND PUMP PULSE WIDTHS.

\begin{tabular}{|c|c|c|c|}
\hline & \multicolumn{3}{|c|}{ Pulse width [ns] } \\
\hline Strain $\left[\mathbf{N}^{\circ}\right.$ Turns $]$ & 10 & 15 & 20 \\
\hline 0 & 0.61 & 0.20 & 0.08 \\
\hline 0.5 & 0.35 & 0.12 & 0.11 \\
\hline 1 & 0.25 & 0.12 & 0.19 \\
\hline 1.5 & 0.3 & 0.18 & 0.13 \\
\hline 2 & 0.22 & 0.14 & 0.14 \\
\hline 2.5 & 0.29 & 0.11 & 0.18 \\
\hline 3 & 0.34 & 0.15 & 0.09 \\
\hline 3.5 & 0.19 & 0.18 & 0.26 \\
\hline 4 & 0.31 & 0.10 & 0.09 \\
\hline
\end{tabular}

Apart from the BOTDA setup, it was also necessary to design and implement a setup enabling to develop the required simultaneous temperature and strain measurements. As it was impossible to perform distributed strain measurements within the climatic chamber, a setup similar to the one described in [22], where a feasibility study of this approach was presented, was chosen. The system (Fig. 5(a)) is an aluminium water tank covered by a thermal insulator material and formed by an air pump (1) used to get an homogeneous water temperature within the tank, an electrical resistance (2) to control the water temperature and two wheels ( 3 and 4$)$ where the hotspot section of the FUT is deployed. Figures 5(b) and (c) show details of the linear positioner used to strain the fiber coiled in the wheels (located under one of these wheels) and the control temperature unit used to established the desired temperature.

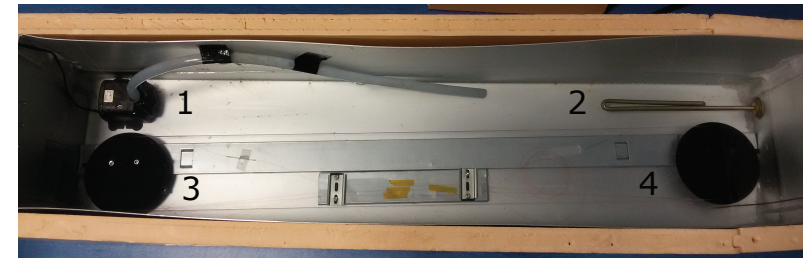

a)

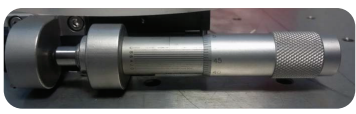

b)

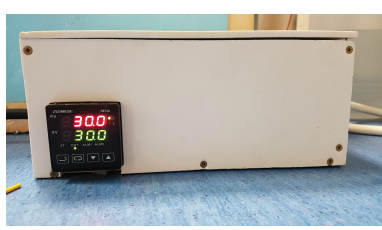

C)

Fig. 5. Setup designed and implemented for the development of the simultaneous temperature and strain measurements: (a) aluminium water tank; (b) linear positioner and (c) temperature control unit.

For the simultaneous temperature/strain tests, a range of temperatures between 22 and $62^{\circ} \mathrm{C}$ (with steps of $10^{\circ} \mathrm{C}$ ) was chosen to facilitate the development of the experiments. The strain range was in this case the one derived from considering 0 to 4 turns (with steps of 0.5 turns) of the linear positioner, thus from 0 to $1536 \mu$ strains. While performing the simultaneous temperature/strain measurements, a study of the BFS error in the hotspot section was performed for a pump pulse width of $20 \mathrm{~ns}$ (chosen for the subsequent analysis) and the results are presented in Table 3. Given
TABLE III

BFS ERROR [MHZ] FOR DIFFERENT TEMPERATURES AND STRAINS AND A PUMP PULSE WIDTH OF 20 NS.

\begin{tabular}{|c|c|c|c|c|c|}
\hline & \multicolumn{5}{|c|}{ Temperature $\left[{ }^{\circ} \mathrm{C}\right]$} \\
\hline Strain [ $\mathbf{N}^{\circ}$ Turns] & 22 & 32 & 42 & 52 & 62 \\
\hline 0 & 4.41 & 5.29 & 5.57 & 5.64 & 5.98 \\
\hline 0.5 & 4.51 & 5.68 & 5.64 & 5.67 & 6.1 \\
\hline 1 & 4.60 & 5.74 & 5.65 & 5.68 & 6.13 \\
\hline 1.5 & 4.75 & 5.69 & 5.76 & 5.90 & 6.35 \\
\hline 2 & 4.68 & 5.69 & 5.85 & 5.93 & 6.49 \\
\hline 2.5 & 4.81 & 5.83 & 5.86 & 5.94 & 6.52 \\
\hline 3 & 4.85 & 5.84 & 5.88 & 5.97 & 6.74 \\
\hline 3.5 & 4.87 & 5.86 & 5.96 & 5.96 & 6.76 \\
\hline 4 & 4.94 & 5.84 & 5.93 & 5.98 & 6.97 \\
\hline
\end{tabular}

the difficulty to obtain homogeneous temperature and strain distributions in our setup, the error was calculated in this case as the mean absolute deviation of the BFS associated with the hotspot section, i.e. the average distance between the BFS associated with each spatial sample within the hotspot and the BFS mean of the whole hotspot section. It can be noticed how for the same strain, the estimated error increases with increasing temperatures, given the difficulty to obtain a completely uniform water temperature distribution within the tank, especially for higher temperatures. It should be mentioned that the FUT is in this case formed by $70 \mathrm{~m}$ of fiber (the length of 1 turn in the strain setup is equivalent to approximately $1.1 \mathrm{~m}$ ), in comparison to the $40 \mathrm{~m}$ FUT used for Tables 1 and 2.

The evolution of the BFS for different strains (0 to 4 turns) at different temperatures $\left(22\right.$ to $\left.52^{\circ} \mathrm{C}\right)$ is depicted in Fig. 6. It can be observed that, in spite of our efforts, the strain in the FUT is not homogeneous, but it exhibits some peaks associated with the manual deployment of the sensing fiber. This fact implies in fact a worse-case scenario for the proposed solution, as the ideal situation for an optimal performance of the ANN would be completely homogeneous BFSs.

\section{REsults}

Once the experimental tests were performed, it was necessary to adapt the acquired data via the pre-processing strategies already introduced in Section II. As already commented, the normalization of the BGS amplitude and the spatial filtering have always been considered, while the remaining three (Lorentzian-Gaussian fit, manual selection of parameters and PCA) have been independently evaluated. After applying PCA to the BOTDA data, 62 PCs were chosen using the criterion of obtaining the best classification rate for the ANN.

Regarding the ANN design, the number of neurons in the input layer will vary depending on the pre-processing strategy considered: 251 input neurons for the Lorentzian-Gaussian fit, 2 for manual selection of parameters and 62 for PCA (same number as chosen PCs). The number of neurons in the hidden layer/s will be modified to analyze the performance 


\begin{tabular}{|llllll|}
\hline \multicolumn{7}{c|}{$\mathrm{N}^{\circ}$ Turns } \\
-0 & -0.5 & -1 & -1.5 & -2 & -2.5 \\
-3 & -3.5 & -4 & & & \\
\hline
\end{tabular}
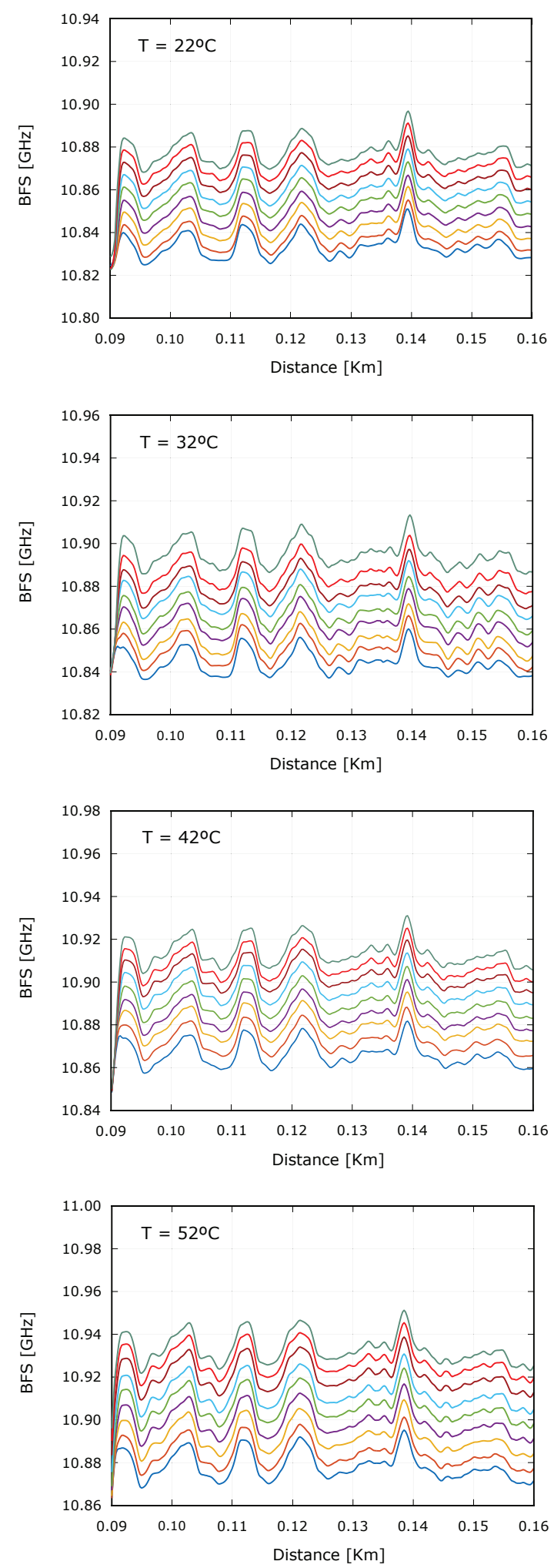

Fig. 6. BFS evolution for simultaneous measurements of temperature and strain.

of the network. The number of output neurons will remain constant at 40 , the number of different classes (temperature and strain pairs) under analysis. The scaled conjugated gradient algorithm with backpropagation has been chosen for the training stage, given its high rate of convergence. A sigmoid activation function has also been considered.

For each pre-processing and ANN configuration, the whole dataset acquired during the experimental tests has been divided into training $(70 \%)$, validation $(15 \%)$ and test $(15 \%)$ datasets. For each considered configuration, the data used for one of these classes have not been shared with the other two classes. After several studies, the best results in terms of the classification performance of the ANN were obtained via PCA, and also considering the normalization and spatial filtering processes described in the previous sections. The employment of the Lorentzian-Gaussian fit gave rise to a classification rate (number of correct associations of an input to its corresponding output over the whole number of inputs evaluated) of about $60 \%$, while the manual selection of the input data to the network allowed to obtain a $70 \%$. The classification results offered via PCA are presented in Table 4, where Neurons indicates the number of neurons in the hidden layer and Training, Validation and Test the corresponding classification results for each stage. The best result is obtained when 80 neurons are used in the hidden layer, offering a classification rate of $88.2 \%$ for the test set. A higher number of neurons in this layer gave rise to and overfitted solution.

TABLE IV

ANN CLASSIFICATION RESULTS VIA PCA.

\begin{tabular}{cccc} 
Neurons & Training [\%] & Validation [\%] & Test [\%] \\
\hline 20 & 86.9 & 77.5 & $\mathbf{7 7 . 1}$ \\
25 & 88.3 & 78.7 & $\mathbf{7 8 . 5}$ \\
30 & 88.1 & 80.4 & $\mathbf{8 0}$ \\
35 & 88.9 & 81.4 & $\mathbf{8 0 . 6}$ \\
40 & 91.3 & 82.2 & $\mathbf{8 2 . 7}$ \\
50 & 93.8 & 83.6 & $\mathbf{8 3 . 8}$ \\
80 & 96.8 & 87.7 & $\mathbf{8 8 . 2}$
\end{tabular}

The analysis of the network cross-entropy (ce) also offers very interesting information about the process. This parameter calculates the difference between the network output and the expected result (target). In this way, the cross-entropy highly penalizes those situations where the output $y$ is far away from the target output $t$. It is calculated as $c e=-t \cdot \log (y)$, and lower values of $c e$ imply a better performance of the network. Figure 7 shows the cross-entropy for the training, validation and test sets when the hidden layer is formed by 80 neurons. It can be observed how the cross-entropy associated with the training set decreases continuously, while this parameter remains constant for the validation and test sets from iteration \#47. This is precisely where the training stage ends, given that the cross-entropy of the validation stage is no longer decreasing. It is also interesting to note that the $c e$-training profile remains close to the other two curves, thus suggesting that there is no overfitting to the training set in this case.

Finally, it is interesting to analyze the results derived from the use of the Lorentzian-Gaussian fit against the raw Brillouin spectra. Although the classification rates are lower in both 


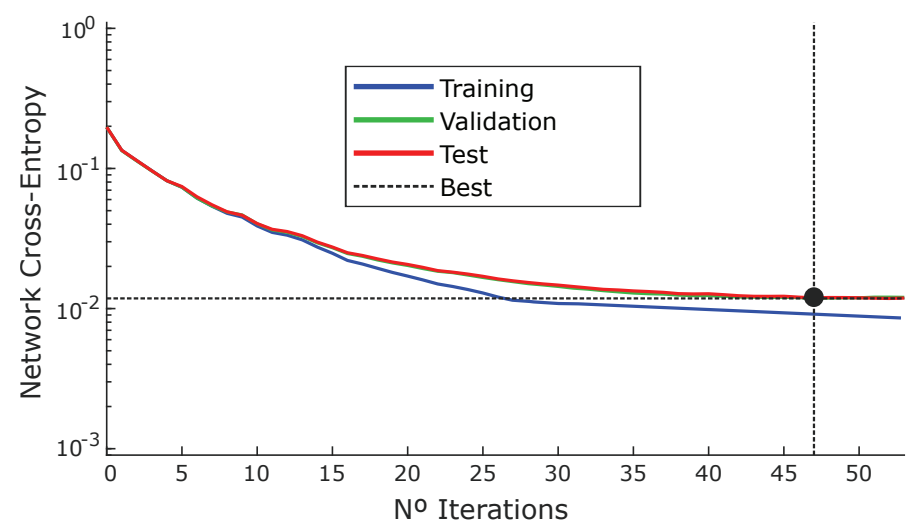

Fig. 7. Network cross-entropy for the best classification result achieved with 80 neurons in the hidden layer.

cases in comparison to those presented in Table 4 using PCA, we noticed that better classification rates (around $80 \%$ ) were achieved when the classical fit to the acquired BGSs was not performed. This was initially surprising considering the starting premise considered in this work; however, an analysis of the intensity noise of the acquired spectra enables a possible explanation. Table 5 presents the variance of the noise associated with the spectra of different pairs of temperature/strain considered, once the gain normalization was carried out. It can be observed how there is no clear trend between temperature and the noise variance, but the latter increases with increasing strain. It seems that the ANN might be using this feature to help in the classification process among the different categories, thus improving the classification results when that information is available (raw spectra) in comparison to the Lorentzian-Gaussian fit approach, where the intensity noise is removed.

TABLE V

NOISE VARIANCE $\left(\sigma^{2}\right)$ FOR THE INTENSITY-NORMALIZED BGSS [A.U. $\cdot 10^{-4}$ ]

\begin{tabular}{|c|c|c|c|c|c|}
\hline & \multicolumn{5}{|c|}{ Temperature $\left[{ }^{\circ} \mathrm{C}\right]$} \\
\hline Strain $\left[\mathbf{N}^{\circ}\right.$ Turns] & 22 & 32 & 42 & 52 & 62 \\
\hline 0.5 & 1.9576 & 1.8351 & 1.9743 & 1.8224 & 1.9871 \\
\hline 1 & 2.1673 & 1.9512 & 2.007 & 1.8938 & 2.0885 \\
\hline 1.5 & 2.2137 & 1.9188 & 2.2070 & 1.9237 & 2.1870 \\
\hline 2 & 2.2256 & 1.9824 & 2.2716 & 1.9418 & 2.3531 \\
\hline 2.5 & 2.2463 & 2.0424 & 2.2187 & 2.1804 & 2.4302 \\
\hline 3 & 2.4432 & 2.1137 & 2.3703 & 2.3396 & 2.5666 \\
\hline 3.5 & 2.4577 & 2.3413 & 2.4951 & 2.4198 & 2.6631 \\
\hline 4 & 2.5295 & 2.3853 & 2.5748 & 2.5391 & 2.6935 \\
\hline
\end{tabular}

\section{CONCLUSION}

In this paper, a new approach for the automatic discrimination of simultaneous temperature/strain measurements in a BOTDA sensor has been presented. The solution is based on the employment of a conventional BOTDA implementation and a processing stage formed by a pre-processing step and an ANN. It has been demonstrated that a classification rate of around $90 \%$ can be achieved, even if the experimental tests have given rise to non-homogeneous BFS profiles associated with the different temperature/strain categories. The best results have been obtained when PCA is used in the pre-processing stage in addition to gain normalization and spatial filtering. It is worth mentioning that this solution does not involve a higher cost or complexity of the required setup. The ANN has been implemented via MATLAB in a conventional desktop PC, achieving real-time processing times. The best classification results are associated with processing times of $2.93 \mathrm{~s}$ for PCA and $92 \mathrm{~s}$ for the ANN training phase (off-line stage), while the whole processing of each new measured BGS (on-line stage) will only take a few milliseconds. Given that the achieved temperature and strain resolutions are clearly lower than those exhibited by previous proposals, future works will be focused on improving this point, as well as the network performance by dealing with the homogeneity of the generated distributed strain and studying the effect of the intensity noise in the classification rate. The system will also be evaluated in field tests.

\section{ACKNOWLEDGMENT}

The authors would like to thank Arianna Picolo for her valuable help during the initial experimental tests and implementation and validation of the ANN solution. This work has been supported by the projects TEC2013-47264-C2-1-R and TEC2016-76021-C2-2-R (AEI/FEDER, UE) and CIBERBBN via FEDER funds.

\section{REFERENCES}

[1] Z. Yang, M. A. Soto, and L. Thévenaz, "200 km fiber-loop brillouin distributed fiber sensor using bipolar golay codes and a three-tone probe," in Proc. of SPIE Vol, vol. 9634, 2015, pp. 96340J-1.

[2] X. Angulo-Vinuesa, S. Martin-Lopez, J. Nuño, P. Corredera, J. D. AniaCastañon, L. Thévenaz, and M. González-Herráez, "Raman-assisted brillouin distributed temperature sensor over $100 \mathrm{~km}$ featuring $2 \mathrm{~m}$ resolution and 1.2 c uncertainty," Journal of Lightwave Technology, vol. 30, no. 8, pp. 1060-1065, 2012.

[3] J. Urricelqui Polvorinos, M. Sagüés García, and A. Loayssa Lara, "Brilloun optical time domain analysis sensor assisted by a brillouin distributed amplifier," in Proc. SPIE 9634, 24th International Conference on Optical Fibre Sensors, $96344 U$ (September 28, 2015). SPIE, 2015.

[4] F. Gyger, E. Rochat, S. Chin, M. Niklès, and L. Thévenaz, "Extending the sensing range of brillouin optical time-domain analysis up to 325 km combining four optical repeaters," in Proc. SPIE, vol. 9157, no. EPFL-CONF-200239. SPIE, 2014, p. 91576Q.

[5] W. Li, X. Bao, Y. Li, and L. Chen, "Differential pulse-width pair botda for high spatial resolution sensing," Optics express, vol. 16, no. 26, pp. $21616-21625,2008$.

[6] R. Ruiz-Lombera, J. Urricelqui, M. Sagues, J. Mirapeix, J. M. LópezHiguera, and A. Loayssa, "Overcoming nonlocal effects and brillouin threshold limitations in brillouin optical time-domain sensors," IEEE Photonics Journal, vol. 7, no. 6, pp. 1-9, 2015.

[7] A. Dominguez-Lopez, Z. Yang, M. A. Soto, X. Angulo-Vinuesa, S. Martin-Lopez, L. Thevenaz, and M. Gonzalez-Herraez, "Reaching the ultimate performance limit given by non-local effects in botda sensors." Society of Photo Optical Instrumentation Engineers (SPIE), 2015.

[8] R. Bernini, A. Minardo, and L. Zeni, "Dynamic strain measurement in optical fibers by stimulated brillouin scattering," Optics letters, vol. 34 , no. 17 , pp. 2613-2615, 2009.

[9] J. Urricelqui, A. Zornoza, M. Sagues, and A. Loayssa, "Dynamic botda measurements based on brillouin phase-shift and rf demodulation," Optics express, vol. 20, no. 24, pp. 26942-26949, 2012.

[10] A. Voskoboinik, O. F. Yilmaz, A. W. Willner, and M. Tur, "Sweep-free distributed brillouin time-domain analyzer (sf-botda)," Optics express, vol. 19, no. 26, pp. B842-B847, 2011.

[11] M. A. Soto and L. Thévenaz, "Modeling and evaluating the performance of brillouin distributed optical fiber sensors," Optics express, vol. 21, no. 25 , pp. 31347-31366, 2013. 
[12] M. A. Soto, J. A. Ramírez, and L. Thévenaz, "Intensifying the response of distributed optical fibre sensors using $2 \mathrm{~d}$ and $3 \mathrm{~d}$ image restoration," Nature communications, vol. 7, 2016.

[13] A. K. Azad, L. Wang, N. Guo, H.-Y. Tam, and C. Lu, "Signal processing using artificial neural network for botda sensor system," Optics express, vol. 24, no. 6, pp. 6769-6782, 2016.

[14] M. Nikles, L. Thevenaz, and P. A. Robert, "Brillouin gain spectrum characterization in single-mode optical fibers," Journal of Lightwave Technology, vol. 15, no. 10, pp. 1842-1851, 1997.

[15] X. Bao, D. J. Webb, and D. A. Jackson, "Combined distributed temperature and strain sensor based on brillouin loss in an optical fiber," Optics Letters, vol. 19, no. 2, pp. 141-143, 1994.

[16] D.-P. Zhou, W. Li, L. Chen, and X. Bao, "Distributed temperature and strain discrimination with stimulated brillouin scattering and rayleigh backscatter in an optical fiber," Sensors, vol. 13, no. 2, pp. 1836-1845, 2013.

[17] C. Lee, P. Chiang, and S. Chi, "Utilization of a dispersion-shifted fiber for simultaneous measurement of distributed strain and temperature through brillouin frequency shift," IEEE Photonics Technology Letters, vol. 13, no. 10, pp. 1094-1096, 2001.

[18] L. Zou, X. Bao, S. Afshar, and L. Chen, "Dependence of the brillouin frequency shift on strain and temperature in a photonic crystal fiber,' Optics letters, vol. 29, no. 13, pp. 1485-1487, 2004.

[19] X. Lu, M. A. Soto, and L. Thévenaz, "Temperature-strain discrimination in distributed optical fiber sensing using phase-sensitive optical timedomain reflectometry," Optics Express, vol. 25, no. 14, pp. 16059 $16071,2017$.

[20] E. Catalano, R. Laiso, R. Bernini, L. Zeni, and A. Minardo, "Simultaneous strain and temperature measurements using dual-wavelength botda," in Optical Fiber Sensors Conference (OFS), 2017 25th. IEEE, 2017, pp. $1-4$.

[21] W. Zou, Z. He, and K. Hotate, "Investigation of strain-and temperaturedependences of brillouin frequency shifts in geo 2-doped optical fibers," Journal of Lightwave Technology, vol. 26, no. 13, pp. 1854-1861, 2008.

[22] R. Ruiz-Lombera, A. Piccolo, L. Rodriguez-Cobo, J. Lopez-Higuera and J. Mirapeix, "Feasibility study of strain and temperature discrimination in a botda system via artificial neural networks," in Optical Fiber Sensors Conference (OFS), 2017 25th. IEEE, 2017, pp. 1-4.

[23] J. Mirapeix, P. García-Allende, A. Cobo, O. Conde, and J. LópezHiguera, "Real-time arc-welding defect detection and classification with principal component analysis and artificial neural networks," $N D T \& e$ International, vol. 40, no. 4, pp. 315-323, 2007.

[24] A. K. Azad, F. N. Khan, W. H. Alarashi, N. Guo, A. P. T. Lau, and C. Lu, "Temperature extraction in brillouin optical time-domain analysis sensors using principal component analysis based pattern recognition," Optics express, vol. 25, no. 14, pp. 16534-16549, 2017.

Ruben Ruiz-Lombera received the degree in Telecommunication Technical Engineering (Electronic Systems) in 2011, the degree in Telecommunication Engineering in 2012, and the Master degree in Information Technologies and Mobile Networks from the University of Cantabria (Spain). He is currently doing his $\mathrm{PhD}$ in the area of distributed fiber sensors, especially those based on Brillouin scattering.

Alberto Fuentes Alberto got his Bachelor's degree in Telecommunications in July 2017 at Univsersity of Cantabria and he is currently enrolled in the Master's degree in Universidad Politecnica de Madrid.

Luis Rodriguez-Cobo received the degree in telecommunication engineering from the University of Cantabria, Spain, in 2009, where since 2009. In 2013 he obtained his $\mathrm{PhD}$. degree in optical sensors for smart structures working at the Photonics Engineering Group (GIF), Department of electronic Technology Industrial Automation and System Engineering. He was also awarded with the $\mathrm{PhD}$ extraordinary award in 2015 for his research carried out during his studies. His research is about applying optical technologies to structural health monitoring systems in different application fields such as energy, industrial applications and environment monitoring.
Jose-Miguel Lopez-Higuera (M93SM98) was born in February 1954, in the village of Ramales de la Victoria, Cantabria, Spain. He obtained the Telecommunication Technical Engineering degree from the Universidad Laboral de Alcala de Henares, Madrid, Spain and the Telecommunication Engineering degree from the Universidad Politecnica de Madrid (UPM), Madrid, Spain. He received the Ph.D. degree in telecommunication engineering, with an extraordinary award, from UPM. He founded and is the Head of the Photonics Engineering Group of the TEISA Department in the University of Cantabria. Currently, he works in the development of Photonics Instrumentation, photonic/optical fibre sensor systems for civil engineering, electrical power, environmental and smart structures and for optical diagnostics for a wide range of applications. He has directed more than $50 \mathrm{R} \& \mathrm{D}$ projects and has written or co-written more than 400 publications in the form of books, chapters of books, papers and conferences, both national and international, and obtained ten patents. He is the Editor and Co-author of several books, including i) "Optical Sensors", UC, 1998; ii) the "Handbook of Photonic Sensing Technology", Wiley and Sons, New York, NY, USA, 2002 and iii) he is the co-editor of the book "Engineering a High-Tech Business: Entrepreneurial experiences and Insights", published by SPIE-Press, USA, 2008. Prof. Lopez-Higuera is Senior Member of the IEEE and member of the IEE, SPIE and OSA.

Jesus Mirapeix received the masters degree in telecommunications engineering in 2000, and the Ph.D. degree with a focus on quality monitoring of welding processes by means of plasma optical spectroscopy, in 2007. Since 2012, he has been an Associate Professor with the Photonics Engineering Group, Department of Electronic Technology, Industrial Automation and System Engineering, University of Cantabria, with teaching and R\&D duties. $\mathrm{He}$ is currently involved in distributed sensor systems based on stimulated Brillouin scattering. He is the Co-Founder of SADIQ Engineering, a company offering products and services for welding, automation, and monitoring. His research interests include fiber optic sensors and processing strategies for online quality assurance of both arc and laser welding processes, with application in the manufacturing of components for the aerospace and nuclear sectors. 